\title{
Dimensional Properties of Polypeptides in the Helix-Coil Transition Region II. Intrinsic Viscosity
}

\author{
Jong Ryul KIM and Taikyue REE \\ Department of Chemistry, Korea Advanced Institute of Science and Technology, \\ P.O. Box 150, Cheongryangni, Seoul 131, Korea
}

(Received February 3, 1984)

\begin{abstract}
The theory presented in the preceidng paper (Part I) for the molecular dimensions, $\left\langle s^{2}\right\rangle_{\mathrm{oI}}$ and $\left\langle s^{2}\right\rangle_{\mathrm{I}}$, of polypeptides in the helix-coil transition region is applied here to determine the intrinsic viscosity $[\eta]_{1}$ (the $[\eta]$ in the transition region) as a function of the helical content $f_{\mathrm{H}}$ which varies with the cooperativity parameter (eq I-16). Using the Kurata-Yamakawa theory, the linear expansion factor $\alpha_{s}$ was converted to the hydrodynamic expansion factor $\alpha_{\eta}$. Thus, with $\alpha_{\eta 1}$ (the $\alpha_{\eta}$ in the transition region) and $\left\langle s^{2}\right\rangle_{\mathrm{ol}}$ (eq I-10), the [ $\left.\eta\right]_{1}$ 's of polypeptides in the helixcoil transition region were calculated (eq 3 ), and compared with experimental results for various kinds of polypeptide-solvent systems with good agreement (Figures 3 and 4).

KEY WORDS Solution Properties / Helix-Coil Transition / Synthetic Polypeptide / Helical Content / Intrinsic Viscosity / Cooperativity Parameter / Kurata-Yamakawa Theory /
\end{abstract}

This paper is a continuation of the preceding one (referred to as Part I, herein) where the expression eq I-10 (eq 10 in Part I), correlating the unperturbed dimension $\left\langle s^{2}\right\rangle_{\mathrm{oI}}$ of polypeptides with helical content $f_{\mathrm{H}}$, was derived, and in which were also presented the equations by which the changing trend of the linear expansion factor $\alpha_{s I}$ in the helix-coil transition region (see eq I-18 and I-20) could be calculated. This paper predicts the intrinsic viscosity $[\eta]_{1}$, i.e., $[\eta]$ in the helix-coil transition region, from $\left\langle s^{2}\right\rangle_{\mathrm{oI}}$ and $\alpha_{\mathrm{SI}} \cdot[\eta]_{\mathrm{I}}$ is of great importance for explaining the behavior of a polypeptide chain in dilute solution with conformation change, but no theoretical attempt has been made along this line. By using $\left\langle s^{2}\right\rangle_{\text {ol }}$ and $\alpha_{\text {sI }}$ with the aid of the Kurata-Yamakawa theory, ${ }^{1}$ which correlates $\alpha_{\mathrm{s}}$ with the hydrodynamic expansion factor $\alpha_{\eta}$, the relation of $[\eta]_{\mathrm{I}} v s . f_{\mathrm{H}}$ is derived in this paper, and compared with experiment, e.g., PBLG in DCA-EDC (ethylene dichloride) ${ }^{2,3}$ and $\operatorname{poly}(\beta$-benzyl L-aspartate) (PBLA) in
DCA- $\mathrm{CHCl}_{3}{ }^{4}$ and in $m$-cresol. ${ }^{4}$

\section{THEORY}

Flory and Fox $^{5}$ proposed the empirical equation

$$
[\eta]=\Phi \frac{\left(6\left\langle s^{2}\right\rangle_{\mathrm{o}}\right)^{3 / 2}}{M} \alpha_{\mathrm{s}}{ }^{3}=\Phi_{\mathrm{o}} \frac{\left(6\left\langle s^{2}\right\rangle_{\mathrm{o}}\right)^{3 / 2}}{M} \alpha_{\eta}{ }^{3}
$$

where $[\eta]$ is the intrinsic viscosity or the limiting viscosity number, $\alpha_{\eta}$, the hydrodynamic expansion factor or the expansion factor for viscosity defined by $\alpha_{\eta}^{3} \equiv[\eta] /[\eta]_{\Theta}\left([\eta]_{\Theta}=[\eta]\right.$ in the unperturbed state), $M$, the molecular weight of the polymer, $\Phi_{0}$, the Flory viscosity constant, a universal constant independent of the nature of polymers, and $\Phi$, the viscosity constant which is a decreasing function $^{5}$ of the excluded volume effect.

$$
\alpha_{\eta}^{3}=\alpha_{s}^{2.43}
$$


Using the second equality of eq 1 and eq I$10,[\eta]_{\mathrm{I}} /[\eta]_{\mathrm{C}}$ may be written as

$$
\frac{[\eta]_{\mathrm{I}}}{[\eta]_{\mathrm{C}}}=\left(\frac{\alpha_{\eta \mathrm{I}}}{\alpha_{\eta \mathrm{C}}}\right)^{3}\left(\frac{1+f_{\mathrm{H}} \cos 80^{\circ}}{1-f_{\mathrm{H}} \cos 80^{\circ}}\right)^{3 / 2}
$$

where $[\eta]_{\mathrm{I}}$ and $[\eta]_{\mathrm{C}}$ are $[\eta]$ for the intermediate chain in the helix-coil transition region and the random-coil, respectively, $\alpha_{\eta \mathrm{I}}$ and $\alpha_{\eta \mathrm{C}}$ are $\alpha_{\eta}$ for the intermediate chain and the randomcoil.

From eq 2 and I-18

$$
\left(\frac{\alpha_{\eta \mathrm{I}}}{\alpha_{\eta \mathrm{C}}}\right)^{3}=\left(\frac{\alpha_{\mathrm{sI}}}{\alpha_{\mathrm{sC}}}\right)^{2.43}=\alpha_{\mathrm{h}}{ }^{2.43}
$$

Substituting eq 4 into 3 leads to

$$
\frac{[\eta]_{\mathrm{I}}}{[\eta]_{\mathrm{C}}}=\alpha_{\mathrm{h}}^{2.43}\left(\frac{1+f_{\mathrm{H}} \cos 80^{\circ}}{1-f_{\mathrm{H}} \cos 80^{\circ}}\right)^{3 / 2}
$$

\section{RESULTS AND DISCUSSION}

\section{Changing Trend of the Expansion Factor}

The quantities, $f_{\mathrm{H}}$ and $[\eta]_{\mathrm{I}} /[\eta]_{\mathrm{C}}$, were calculated from eq I-16 and 5, respectively, by eq I14 and I-15 for $n=950$ at various given $f$ with $\sigma=2.0 \times 10^{-4.8}$ The results are presented in Table I. In this table, it may be noted that $[\eta]_{\mathrm{I}}$ increases slowly over the range of $f_{\mathrm{H}}$ from zero to about 0.85 , but steeply from $f_{\mathrm{H}} \simeq 0.85$ to 1.0 . This is due to the changing trend of $\alpha_{\mathrm{sI}}$ with $f_{\mathrm{H}}$ (see Table I of Part I and Figure 1). That is, $[\eta]_{\mathrm{I}} /[\eta]_{\mathrm{C}}$ is a function of $\alpha_{\mathrm{h}}$ (eq 5) which equals $\alpha_{\mathrm{sI}} / \alpha_{\mathrm{sC}}$ (eq 4) and behaves as shown in Figure 1.

Values of $\alpha_{\mathrm{sI}} / \alpha_{\mathrm{SC}}\left(=\alpha_{\mathrm{h}}\right)$ over the whole range of $f_{\mathrm{H}}$ for $n=950$ with the values of $\sigma=$ $0.5 \times 10^{-4}, 1.0 \times 10^{-4}$, and $2.0 \times 10^{-4}$, were calculated from eq I-20 in collaboration with eq I-14 to I-16, and the results are shown in Figure 1. The quantity $\Phi$ was obtained from the first equality of eq 1 using the data of $[\eta]$ and $\left\langle s^{2}\right\rangle\left(=\alpha_{s}^{2}\left\langle s^{2}\right\rangle_{o}\right)$; it generally decreased $^{6}$ with increasing $\alpha_{\mathrm{s}}$ in accordance with the relation $\Phi=\Phi_{\mathrm{o}} \times \alpha_{\mathrm{s}}^{-0.57}$ obtained from eq 1 and 2. Norisuye ${ }^{3}$ determined $\Phi$ of two high-molecular weight PBLG samples in DCA containing $8.3 \mathrm{wt} \%$ of $\mathrm{CHL}$ in the thermal
Table I. Dependence of $f_{\mathrm{H}}$ and $[\eta]_{\mathrm{l}} /[\eta]_{\mathrm{C}}$ on $f$ for the chain of $n=950$ taking $\sigma=2.0 \times 10^{-4}$

\begin{tabular}{ccc}
\hline$f$ & $f_{\mathrm{H}}$ & {$[\eta]_{\mathrm{I}} /[\eta]_{\mathrm{C}}$} \\
\hline 0.050 & 0.048 & 1.0255 \\
0.150 & 0.142 & 1.0768 \\
0.250 & 0.234 & 1.1297 \\
0.350 & 0.325 & 1.1848 \\
0.450 & 0.417 & 1.2429 \\
0.525 & 0.486 & 1.2889 \\
0.600 & 0.556 & 1.3373 \\
0.675 & 0.628 & 1.3888 \\
0.750 & 0.702 & 1.4439 \\
0.825 & 0.778 & 1.5038 \\
0.875 & 0.832 & 1.5472 \\
0.900 & 0.860 & 1.6972 \\
0.925 & 0.889 & 2.0110 \\
0.950 & 0.919 & 2.5055 \\
0.975 & 0.952 & 3.4955 \\
\hline
\end{tabular}

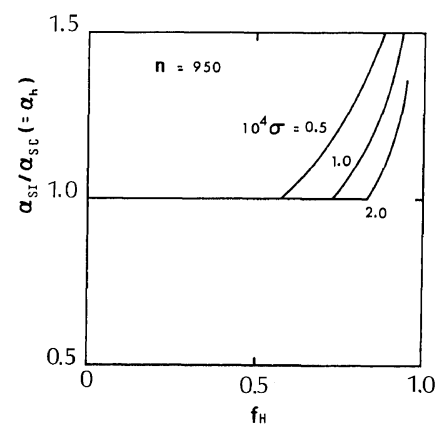

Figure 1. Dependence of the expansion factor $\alpha_{\mathrm{SI}}$ on the helical content $f_{\mathrm{H}}$ for $n=950$ with $\sigma=0.5 \times 10^{-4}$, $1.0 \times 10^{-4}$, and $2.0 \times 10^{-4}$.

helix-coil transition region. The value of $\sigma$ of the polypeptide-solvent system was evaluated ${ }^{7}$ as $0.72 \times 10^{-4}$. According to Norisuye, ${ }^{3} \Phi$ is almost constant in the region of $0 \leq f_{\mathrm{H}}<$ about 0.7 , and decreases sharply as $f_{\mathrm{H}}$ approaches unity. Combining this experimental fact with the above $\Phi v s . \alpha_{\mathrm{s}}$ relation, it is evident that $\alpha_{\mathrm{sI}}$ is almost constant in the region from $f_{\mathrm{H}}=0$ to about 0.7 , but sharply increases in the region of $f_{\mathrm{H}}>0.7$ for the system of $\sigma=0.72 \times 10^{-4}$. This is in good agreement with the results shown in Figure 1.

The cooperativity parameter $\sigma$ has an important effect on $\alpha_{\mathrm{SI}}$ as shown in Figure 1, from 


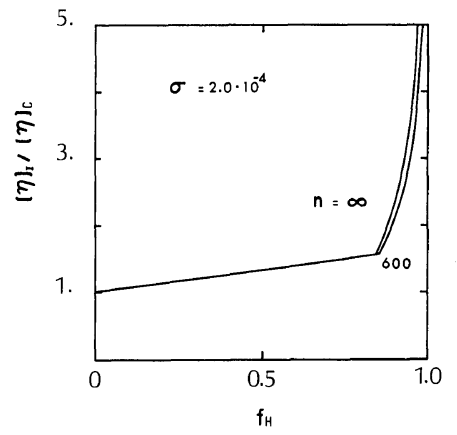

Figure 2. Dependence of $[\eta]_{\mathrm{I}} /[\eta]_{\mathrm{C}}$ on the helical content $f_{\mathrm{H}}$ with $\sigma=2.0 \times 10^{-4}$ for $n=\infty$ and 600 .

which it can be seen that a smaller $\sigma$ gives a larger $\alpha_{\mathrm{sI}}$ at a given helical content $f_{\mathrm{H}}$. This is understandable since a smaller $\sigma$ yields a smaller $g_{\mathrm{H}}$ (the average number of helical sequences, eq I-14) and produces a larger $n_{\mathrm{h}}$ (the average number of residues per helical sequence, see eq I-15) in the chain for fixed $f_{\mathrm{H}}$ and $n$. Consequently, $\alpha_{\mathrm{h}}\left(=\alpha_{\mathrm{sl}} / \alpha_{\mathrm{sC}}\right)$ becomes larger according to eq I-20. This reasonably explains the data shown in Figure 1.

The values of $[\eta]_{\mathrm{I}} /[\eta]_{\mathrm{c}}$ for $n=950$ were calculated from eq 5, and are shown in Table $I$ as previously mentioned. By a similar method used for Table $\mathrm{I}$, the values of $[\eta]_{\mathrm{I}} /[\eta]_{\mathrm{C}}$ and $f_{\mathrm{H}}$ were calculated for $n=600$, and the results are shown in Figure 2. For $n=\infty$, eq I-21 was used with eq 5 . The calculated results for $n=$ $\infty$ also appear in Figure 2. Figure 2 shows that the dependence of $[\eta]_{\mathrm{I}} /[\eta]_{\mathrm{C}}$ on the chain length $n$ is small; i.e., $\alpha_{\mathrm{h}}\left(\right.$ or $\left.\alpha_{\mathrm{sl}} / \alpha_{\mathrm{sC}}\right)$ does not depend very much on $n$ (see eq 4 and 5). This is quite different from the effect of $\sigma$, which yields a great difference as shown in Figure 1.

\section{Comparison with Experiment in the Transition Region}

Teramoto et al. ${ }^{2}$ measured $[\eta]_{\mathrm{I}}$ vs. $f_{\mathrm{H}}$ for several samples of PBLG in DCA-EDC mixed solvents of different composition. Their experimental $[\eta]_{\mathrm{I}}$ values $v s . f_{\mathrm{H}}$ for the samples of $\bar{N}_{w}=1830$ and 1150 are shown in Figure 3.

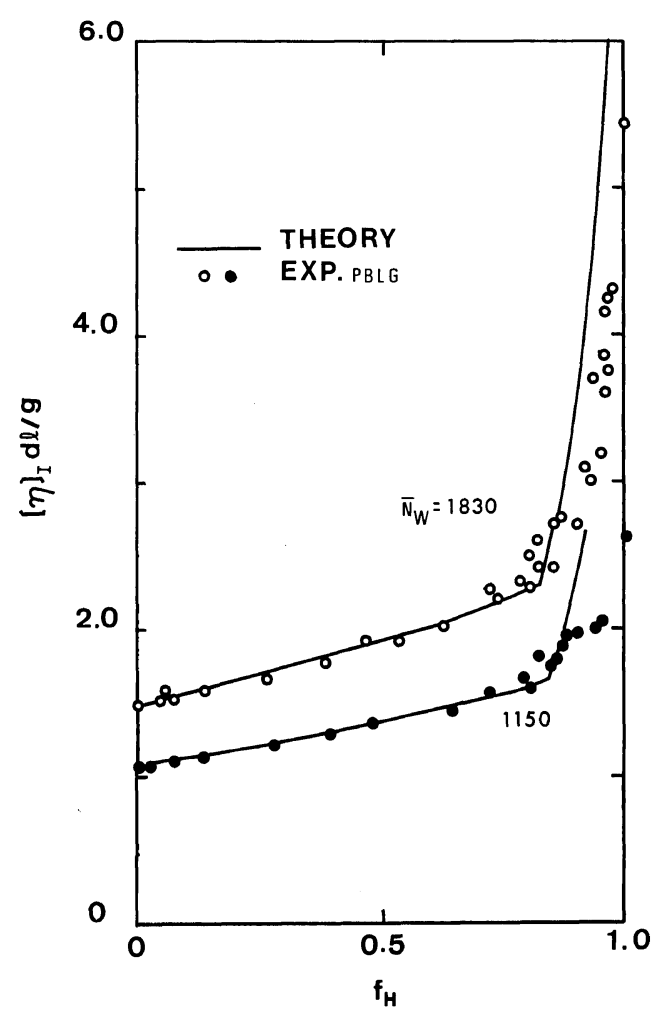

Figure 3. Variation in $[\eta]_{\mathrm{I}}$ with the helical content $f_{\mathrm{H}}$. The experimental points ${ }^{2}$ are for PBLG $\left(\bar{N}_{w}=1830\right.$ and $1150)$ in DCA-EDC mixtures.

Zimm and $\mathrm{Bragg}^{8}$ obtained $\sigma=2.0 \times 10^{-4}$ by a curve-fitting method using the optical rotation data in the helix-coil transition region of PBLG with various molecular weights in DCA-EDC mixed solvents. We calculated $[\eta]_{\mathrm{I}} /$ $[\eta]_{\mathrm{C}}$ from eq 5 using $\sigma=2.0 \times 10^{-4}$, and applying the method used for obtaining Table I. Our results are shown by full curves in Figure 3 , where the values of $[\eta]_{\mathrm{C}}$ are equal to 1.49 and $1.01 \mathrm{dl} \mathrm{g}^{-1}$ (Teramoto et al.'s data) for $\bar{N}_{w}=1830$ and 1150 , respectively. The experimental $[\eta]_{\mathrm{I}}$ values are compared with our theoretical values in Figure 3 with good agreement. Norisuye ${ }^{3}$ repeated the same kind of experiment for PBLG samples with the chain lengths different from Teramoto et al.' $\mathrm{s}^{2}$ in mixed solvents of DCA and EDC. Norisuye's data exhibit almost the same behavior 


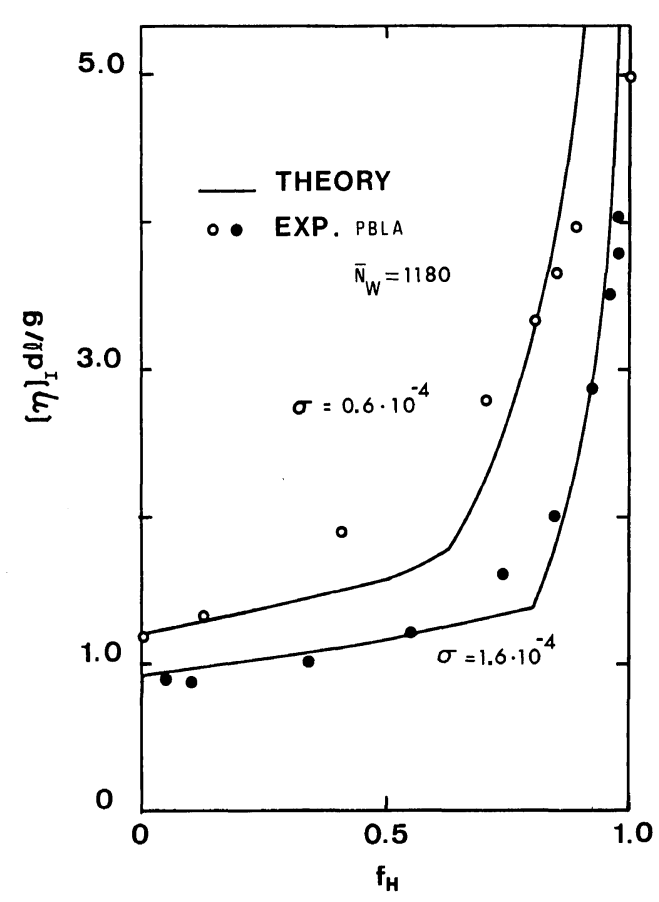

Figure 4. Variation in $[\eta]_{\mathrm{I}}$ with the helical content $f_{\mathrm{H}}$ for PBLA $\left(\bar{N}_{w}=1180\right)$. Experimental points: ${ }^{4}$ open circles, data for a mixed solvent DCA-CHCl (5.7 vol\% DCA); filled circles, for $m$-cresol.

of $[\eta]_{\mathrm{I}} /[\eta]_{\mathrm{C}}$ vs. $f_{\mathrm{H}}$ as Teramoto et al.'s shown in Figure 3.

Hayashi et al. ${ }^{4}$ obtained experimental data of $[\eta]_{\mathrm{I}} v s . f_{\mathrm{H}}$ for PBLA of $\bar{N}_{w}=1180$ in $m$-cresol and in a mixture of chloroform and DCA ( $5.7 \mathrm{vol} \% \mathrm{DCA})$. The cooperativity parameter was distinctly smaller in the latter $(\sigma=$ $\left.0.6 \times 10^{-4}\right)^{4}$ than former solvent $(\sigma=$ $\left.1.6 \times 10^{-4}\right){ }^{4}$ Their experimental results are shown in Figure 4, where the data clearly exhibit the dependence of the relation of $[\eta]_{\mathrm{I}} v s$. $f_{\mathrm{H}}$ on $\sigma$. Our calculated results from eq 5 with $\sigma=0.6 \times 10^{-4}$ and $[\eta]_{\mathrm{C}}=1.30 \mathrm{dl} \mathrm{g}^{-1}$ (both
Hayashi et al.'s data) for PBLA in a mixture of $\mathrm{CHCl}_{3}$ and DCA $(5.7 \mathrm{vol} \%)$ are shown in Figure 4 , where the $\alpha_{\mathrm{h}}$ factor in eq 5 is calculated from eq I-20 in collaboration with eq I-14 to I-16. In Figure 4, our results calculated with

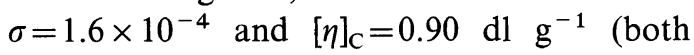
Hayashi et al.'s data ${ }^{4}$ ) for PBLA in $m$-cresol are shown by a full curve. The agreement between our theoretical results and the experimental data is fairly good, and indicates the correct prediction of the $\sigma$ effect on the behavior of $[\eta]_{\mathrm{I}} v s . f_{\mathrm{H}}$.

Acknowledgement. We acknowledge with appreciation the Korea Research Center for Theoretical Physics and Chemistry for a partial support of this work.

\section{REFERENCES}

1. (a) M. Kurata and H. Yamakawa, J. Chem. Phys., 29, 311 (1958); (b) H. Yamakawa, "Modern Theory of Polymer Solution," Harper \& Row, New York, N.Y., 1971, p 302.

2. A. Teramoto, K. Nakagawa, and H. Fujita, J. Chem. Phys., 46, 4197 (1967).

3. (a) T. Norisuye, Thesis, Osaka University, 1973; (b) A. Teramoto and H. Fujita, Adv. Polym. Sci., 18, 65 (1975).

4. Y. Hayashi, A. Teramoto, K. Kawahara, and H. Fujita, Biopolymers, 8, 403 (1969).

5. P. J. Flory, "Principles of Polymer Chemistry," Cornell University Press, New York, N.Y., 1953, pp $611-612$; H. Yamakawa, "Modern Theory of Polymer Solution," Haper \& Row, New York, N.Y., 1971, pp 299-309.

6. H. Yamakawa, "Modern Theory of Polymer Solution," Harper \& Row, New York, N.Y., 1971, pp 379-382.

7. T. Norisuye, A. Teramoto, and H. Fujita, Polym. J., 4, 323 (1973).

8. B. H. zimm and J. K. Bragg, J. Chem., Phys., 31, 526 (1959). 